\title{
Comunicação
}

[Communication]

\section{Freqüência de Giardia spp. por duas técnicas de diagnóstico em fezes de cães}

[Frequency of Giardia spp. for two diagnosis methods in feces of dogs]

\author{
M.J.S. Mundim ${ }^{1}$, S.Z. Souza ${ }^{2}$, S.M. Hortêncio ${ }^{2}$, M.C. Cury ${ }^{1}$ \\ ${ }^{1}$ Laboratório de Parasitologia - Universidade Federal de Uberlândia \\ Avenida Pará, 1720, Bloco 4C \\ Campus Umuarama \\ 38400-902 - Uberlândia, MG \\ ${ }^{2}$ Faculdade de Medicina Veterinária - UFU
}

Recebido para publicação em 26 de setembro de 2002

Recebido para publicação, após modificações, em 20 de maio de 2003

E-mail: cury@umuarama.ufu.br

Dos protozoários que freqüentemente acometem os animais e o homem, Giardia spp. tem despertado maior interesse dos pesquisadores, possivelmente por seu potencial como agente de zoonose, além de causar, em animais jovens, diarréia intermitente com comprometimento da digestão e absorção de alimentos, acarretando desidratação, perda de peso e morte.

As espécies de Giardia isoladas de mamíferos apresentam aspectos morfológicos e propriedades antigênicas, genéticas e bioquímicas similares (Leib, Zajac, 1999).

Sua prevalência, particularmente nos cães, apresenta índices variáveis, dependendo da localização geográfica, do método utilizado para o diagnóstico e da população estudada (Collins et al., 1987; Nikolic et al., 1993; Marcel et al., 1994). Há maior sensibilidade de animais menores de um ano de idade do que adultos, sugerindo o desenvolvimento de certo grau de resistência com o aumento da idade. Os animais de rua ou aqueles densamente abrigados (canis e lojas) estão mais expostos, devido ao maior contato com água, alimentos e fezes de animais ou de pessoas contaminadas (Kirkpatrick, Farrel, 1984).

As várias cepas de Giardia possuem grau variado de patogenicidade. Os sinais clínicos podem ser autolimitantes em alguns pacientes e a doença grave ocorre em filhotes e em animais com doenças concomitantes ou debilitados, sendo a diarréia o sintoma mais comum.

O método de diagnóstico considerado mais eficaz para a giardíase é a técnica de centrífugo-flutuação em solução de sulfato de zinco a 33\% (densidade 1.180). Entretanto outras técnicas, como a centrífugosedimentação com mertiolato-iodo-formaldeído, têm sido utilizadas com bons resultados (Zimmer, Burrington, 1986).

Este estudo teve como objetivo determinar a freqüência de Giardia spp. em cães provenientes de canis da cidade de Uberlândia, usando a centrífugo-flutuação com sulfato de zinco e a centrífugo-sedimentação com mertiolato-iodo-formaldeído (MIFC).

Foram colhidas, ao acaso, 100 amostras de fezes de cães machos e fêmeas, de diferentes raças, com idades entre dois meses a sete anos e provenientes de cinco canis de Uberlândia, Minas Gerais. 
De cada animal foram colhidas três amostras com intervalos de sete dias entre cada colheita. As amostras de fezes foram colhidas sempre no período da manhã e processadas pelos métodos de centrífugoflutuação em solução de sulfato de zinco a 33\% (Faust et al., 1938) e centrífugo-sedimentação pelo mertiolato-iodo-formaldeido (MIFC) (Blagg et al., 1955). Foram examinadas para cada método duas lâminas de cada amostra, perfazendo 1.200 lâminas.

As freqüências obtidas segundo o sexo e a idade foram avaliadas estatisticamente pelo teste de dispersão de freqüência (qui-quadrado) e para comparar os métodos laboratoriais utilizou-se o teste binominal para diferença de proporções, ambos com 5\% de probabilidade (Triola, 1999).

Dos 100 cães examinados, 41,0\% apresentavam-se positivos para Giardia spp., independente do método utilizado. A freqüência encontrada pode ser considerada muito elevada frente aos trabalhos de Sogayar e Corrêa (1984) e Lopes et al. (2001) que demonstraram índices de infecção de 3,4\% e 6,6\%, respectivamente. Na giardíase o contato direto animal-animal facilita a transmissão e nos canis o maior número de animais em um espaço limitado pode facilitar a contaminação, sendo essa a possível explicação para a freqüência tão elevada.

Do total de animais examinados, 28 (28,0\%) apresentaram diarréia e desses 15 (53,6\%) estavam infectados por Giardia spp e em apenas um havia associação com Dipylidium caninum. Lopes et al. (2001) compararam o índice de positividade e a existência de diarréia, observando apenas 3,4\% de concomitância entre eles. Isso porque a maioria das infecções pelo protozoário é assintomática. Entretanto, nos animais que apresentam sintomas a diarréia é o achado mais freqüente.

A freqüência de positivos foi maior $(\mathrm{P}<0,05)$ nos animais com até 12 meses de idade (Tab. 1). Estes dados são condizentes com os de Kirkpatrick (1987) e Leib e Zajac (1999) que relataram maior freqüência de Giardia spp. em cães e gatos com idade inferior a três anos, em especial entre aqueles com até 12 meses. O papel da imunidade no curso da infecção requer maiores esclarecimentos, e o que se observa é uma maior sensibilidade de animais jovens por apresentar, possivelmente, sistema imunológico não totalmente amadurecido. Considera-se ainda que o comportamento dos filhotes facilita a contaminação, já que eles têm contato mais freqüente com todo o tipo de material que pode estar contaminado com cistos de Giardia spp.

Tabela 1. Freqüência de Giardia spp. em 100 cães, machos e fêmeas, procedentes de canis de Uberlândia, Minas Gerais, de acordo com a faixa etária

\begin{tabular}{|c|c|c|c|c|c|c|c|c|c|}
\hline \multirow[b]{2}{*}{ Faixa etária } & \multicolumn{3}{|c|}{ Machos } & \multicolumn{3}{|c|}{ Fêmeas } & \multicolumn{3}{|c|}{ Total } \\
\hline & $\mathrm{N}$ & $\mathrm{P}$ & $\%$ & $\mathrm{~N}$ & $\mathrm{P}$ & $\%$ & $\mathrm{~N}$ & $\mathrm{P}$ & $\%$ \\
\hline$<12$ meses & 12 & 7 & 58,3 & 26 & 19 & 73,0 & 38 & 26 & 68,4 \\
\hline$>12$ meses & 23 & 4 & 17,4 & 39 & 11 & 28,2 & 62 & 15 & 24,2 \\
\hline Total & 35 & 11 & 31,4 & 65 & 30 & 46,1 & 100 & 41 & 41,0 \\
\hline
\end{tabular}

$\mathrm{N}=$ número de animais, $\mathrm{P}=$ positivos

Não houve diferença entre sexos quanto à freqüência de cistos de Giardia spp. nas fezes (Tab. 1). Hoskins et al. (1982) e Kirkpatrick (1987) também não encontraram relação entre sexo ou raça e positividade para Giardia spp.

Não se observou diferença $(\mathrm{P}>0,05)$ entre métodos de processamento das fezes quanto à freqüência de cistos. Tanto o MIFC quanto o sulfato de zinco demonstraram a mesma eficiência na detecção do parasita (Tab. 2).

O método de MIFC foi o que detectou maior número de casos, apresentando 38,0\% de positividade. Zimmer e Burrington (1986) citaram que a prevalência da giardíase em cães com ou sem sintomas, na América do Norte, variou de $0,6 \%$ a 60,0\%. A disparidade nesses resultados é atribuída, em parte, às 
diferenças nas técnicas de diagnóstico utilizadas. Em medicina veterinária, o exame das fezes tem demonstrado ser o meio mais prático e eficiente de diagnóstico da giardíase. Na literatura, o método do sulfato de zinco é considerado o mais viável, pela sensibilidade e baixo custo. Neste estudo, o MIFC detectou maior número de amostras positivas, provavelmente por fornecer maior quantidade de sedimento para análise, aumentando a probabilidade de detecção do protozoário. Entretanto, Zimmer e Burrington (1986) consideram esse método inadequado para o diagnóstico da giardíase, pois os cistos do protozoário não são facilmente visualizados em meio aos detritos fecais.

Tabela 2. Freqüência de Giardia spp. em 100 amostras de fezes de cães procedentes de canis de Uberlândia, Minas Gerais, conforme o método de diagnóstico e o período de colheita

\begin{tabular}{lccccc}
\hline \multirow{2}{*}{$\begin{array}{l}\text { Número de } \\
\text { colheitas }\end{array}$} & \multicolumn{2}{c}{ Sulfato de zinco } & & \multicolumn{2}{c}{ MIFC } \\
\cline { 2 - 3 } \cline { 5 - 6 } & $\mathrm{P}$ & $\%$ & & $\mathrm{P}$ & $\%$ \\
\hline Primeira & 15 & 15,0 & & 22 & 22,0 \\
Segunda & 22 & 22,0 & & 34 & 34,0 \\
Terceira & 29 & 29,0 & & 38 & 38,0 \\
Total & 29 & 29,0 & & 38 & 38,0 \\
\hline \multicolumn{2}{l}{ MIFC= centrífugo-sedimentação pelo mertiolato-iodo-formaldeido; P= positivo. }
\end{tabular}

Em relação ao número de colheitas, observou-se que quanto maior o seu número maior a chance de se obter amostras positivas. De acordo com Heymans et al. (1987) e Adam (1991), é recomendado que se faça o exame em três amostras consecutivas colhidas em dias alternados, pois os cistos são excretados intermitentemente, evitando assim falsos negativos.

Este foi o primeiro estudo realizado em Uberlândia, MG. Em suma, cães procedentes de canis da cidade apresentam elevada freqüência de cistos de Giardia spp. nas fezes, principalmente nos animais com até 12 meses de idade, não havendo diferenças de ocorrência entre sexos e raças. As técnicas de diagnóstico comportaram-se igualmente na detecção dos cistos, com leve (não significativa) supremacia para a do MIFC.

Palavras-chave: Giardia sp., giardíase, cão, sulfato de zinco, MIFC

\begin{abstract}
One hundred fecal samples from male and female dogs of several ages and breeds were collected in kennels of Uberlândia in Minas Gerais, Brazil. These samples were analyzed to determine the frequency of Giardia spp. using two different diagnostic methods: zinc sulfate flotation technique and merthiolateiodine-formaldehyde concentration (MIFC). The frequency of giardiasis was 41\%. Dogs, which were less than 12 months of age, were the most parasitized (68.4\%). No difference between male and female frequency of giardiasis (31.4\% and $46.1 \%$, respectively) was observed. MIFC detected $38 \%$ of positive samples and zinc sulfate flotation technique $29 \%$. Giardia spp. is present in dogs of Uberlândia's kennels in a high frequency.
\end{abstract}

Keywords: Giardia spp., giardiasis, dog, zinc sulfate flotation technique, MIFC

\title{
AGRADECIMENTOS
}

Os autores agradecem à técnica do laboratório de parasitologia da Universidade Federal de Uberlândia, Elaine Silva Marques Faria, pelo auxílio no processamento das análises das amostras de fezes.

\section{REFERÊNCIAS BIBLIOGRÁFICAS}


ADAM, R.D. The biology of Giardia spp. Microbiol. Rev., v.55, p.706-732, 1991.

BLAGG, W.S.; SCHLOEGEL, E.L.; MANSOUR, N.S. et al. A new concentration technic for demonstration of protozoa and helminth eggs in feces. Am. J. Trop. Med. Hyg., v.4, p.23-28, 1955.

COLLINS, G.H.; POPE, S.E.; GRIFFIN, D.L. et al. Diagnosis and prevalence of Giardia spp. in dogs and cats. Aust. Vet. J., v.64, p.89-90, 1987.

FAUST, E.C.; D'ANTONI, I.C.; ODON, V. et al. A critical study of clinical laboratory technics for the diagnosis of protozoan cysts and helminths eggs in feces. I. Preliminary communication. Am. J. Trop. Med., v.18, p.169-183, 1938.

HEYMANS, H.S.A.; ARONSON, D.C.; VAN HOOFT, M.A.J. Giardiasis in childhood: an unnecessarily expensive diagnosis. Eur. J. Paed., v.146, p.401-403, 1987.

HOSKINS, J.D.; MALONE, J.B.; SMITH, P.H. Prevalence of parasitism diagnosed by fecal examination in Louisiana dogs. Am. J. Vet. Res., v.43, p.1106-1109, 1982.

KIRKPATRICK, C.E. Giardiasis. Vet. Clin. North Am.: Small Anim. Pract., v.17, p.1377-1387, 1987.

KIRKPATRICK, C.E.; FARREL, J.P. Feline giardiasis: observations on natural and induced infections. Am. J. Vet. Res., v.45, p.2182-2188, 1984.

LEIB, M.S.; ZAJAC, A.M. Giardiasis infection in dogs and cats. Vet. Med., v.94, p.793-802, 1999.

LOPES, R.S.; SANTOS, K.R.; TAKAHIRA, R.K. et al. Ocorrência de giardíase em cães e gatos no município de Botucatu-SP. J. Bras. Patol., v.37, p.224, 2001 (Resumos).

MARCEL, A.M.; MANSO, E.O.; PÊREZ, H.S. et al. Frecuencia de giardiasis en algunas especies de animales domésticos de la provincia de Villa Clara, Cuba. Vet. Méx., v.25, p.337-340, 1994.

NIKOLIC, A.; KULISIC, Z.; BOJKOVSKI, J. Giardiasis as a zoonosis: the prevalence of Giardia in dogs in Belgrade. Acta Vet., (Belgrade), v.43, p.239-243, 1993.

SOGAYAR, M.I.L.; CORRÊA, F.M.A. Giardia in dogs in Botucatu, São Paulo State, Brazil: a comparative study of canine and human species. Rev. Ciên. Biomed. São Paulo, v.5, p.69-73, 1984.

TRIOLA, M.F. Introdução a estatística. 7.ed. Rio de Janeiro: LTC, 1999. 410p.

ZIMMER, J.F.; BURRINGTON, D.B. Comparison of four technics of fecal examination for detecting canine giardiasis. J. Am. Anim. Hosp. Assoc., v.22, p.161-167, 1986. 